\title{
Current neurosurgical management and the impact of the extent of resection in the treatment of malignant gliomas of childhood: a report of the Children's Cancer Group Trial No. CCG-945
}

Jeffrey H. Wisoff, M.D., James M. Boyett, Ph.D., Mitchel S. Berger, M.D., Catherine Brant, B.A., Hao Li, M.S., Allan J. Yates, M.D., Ph.D., C.P.N.P., Patricia McGuire-Cullen, R.N., M.S., Patrick A. Turski, M.D., Leslie N. Sutton, M.D., Jeffrey C. Allen, M.D., Roger J. Packer, M.D., and Jonathan L. Finlay, M.B.,Ch.B.

Divisions of Pediatric Neurosurgery and Pediatric Neuro-Oncology, New York University Medical Center, New York, New York; Department of Biostatistics, St. Jude Children's Research Hospital, Memphis, Tennessee; Division of Neurosurgery, Children's Hospital and Medical Center, Seattle, Washington; Department of Pediatric Oncology, Memorial Sloan-Kettering Cancer Center, New York, New York; Division of Neuropathology, Ohio State University, Columbus, Ohio; Department of Pediatrics, Presbyterian/St. Luke's Medical Center, Denver, Colorado; Department of Neuro-Radiology, University of Wisconsin, Madison, Wisconsin; Division of Neurosurgery, Children's Hospital of Philadelphia, Philadelphia, Pennsylvania; and Department of Neurology, Children's National Medical Center, Washington, D.C.

Object. One hundred seventy-two children with high-grade astrocytomas were treated by members of the Children's Cancer Group in a prospective randomized trial designed to evaluate the role of two chemotherapy regimens. Seventy-six percent of the patients (131 children) in whom a diagnosis of either anaplastic astrocytoma or glioblastoma multiforme was confirmed by central pathological review are the subject of this report.

Methods. Patients were stratified according to the extent of tumor resection (biopsy $[<10 \%]$, partial resection [10-50\%], subtotal resection [51-90\%], near-total resection [> 90\%], and total resection) as determined by surgical observation and postoperative computerized tomography scanning. Information on contemporary neurosurgical management was obtained from the patient's operative records and standardized neurosurgical report forms.

The vast majority of tumors were supratentorial: $63 \%$ (83 tumors) in the superficial cerebral hemisphere, $28 \%$ (37 tumors) in the deep or midline cerebrum, and only $8 \%$ (11 tumors) in the posterior fossa. A significant association was detected between the primary tumor site and the extent of resection $(\mathrm{p}<$ 0.0001 ). A radical resection (>90\%) was performed in $37 \%$ of the children: $49 \%$ of the tumors in the superficial hematoma and $45 \%$ of tumors in the posterior fossa compared with $8 \%$ of midline tumors. Tumor location could also be used to predict the need for both temporary and permanent cerebrospinal fluid (CSF) diversion. Half of the deep tumors and $8 \%$ of the hemispheric astrocytomas ultimately required a permanent CSF shunt. 
Improvement in preoperative neurological deficits and level of consciousness was seen in $36 \%$ and $34 \%$ of the children, respectively. New or increased deficits were present in $14 \%$ of the children, with $6 \%$ experiencing a diminished sensorium after surgery. Postoperative nonneurological complications were rare, infection, embolization, and CFS fistula each occurring in $1.7 \%$ of the children.

Univariate and multivariate analyses demonstrated that radical tumor resection (>90\%) was the only therapeutic variable that significantly improved progression-free survival (PFS) rates. For all patients with malignant astrocytomas, the distributions of PFS rates were significantly different $(p=0.006)$ following radical resection compared with less extensive (3/4 90\%) resection. The 5-year PFS rates were $35 \pm 7 \%$ and $17 \pm 4 \%$, respectively. The differences in the distribution of PFS rate were significantly different for the subsets of patients with anaplastic astrocytoma $(\mathrm{p}=0.055)$ and glioblastoma multiforme $(\mathrm{p}=0.046)$. The 5-year PFS rates for anaplastic astrocytoma were $44 \pm 11 \%$ and $22 \pm 6 \%$ for cases in which the tumor was radically resected and less than radically resected, respectively; whereas the 5-year PFS rates for glioblastoma multiforme were $26 \pm 9 \%$ and $4 \pm 3 \%$ for cases in which the tumor was radically resected and less than radically resected, respectively.

Conclusions. The demonstration of a survival advantage provided by radical resection should prompt neurosurgeons to treat malignant pediatric astrocytomas with aggressive surgical resection prior to initiation of radiotherapy or adjuvant chemotherapy.

\section{Key Words * anaplastic astrocytoma * brain neoplasm * extent of tumor resection * glioblastoma multiforme * survival rate * children}

Central nervous system tumors are the second most common form of neoplasia to affect the pediatric population and the third leading cause of death in children younger than 16 years of age.[29,43,45] There are approximately 1800 new pediatric brain tumors in the United States annually, with malignant gliomas constituting $6.5 \%$ of the intracranial neoplasms of childhood.[1,5,13,14,43] These tumors have traditionally carried a poor prognosis, similar to adult tumors. Children with glioblastomas multiforme (GBM) have a dismal outcome, with 5-year survival rates ranging from 5 to $15 \%$.[14,34,40,46] Children who have anaplastic astrocytoma (AA) fare better, with a 20 to $40 \%$ long-term rate of survival.[34,40,46] Leptomeningeal dissemination has been noted to occur in approximately 5 to $20 \%$ of children with malignant astrocytomas, especially in those with tumors located adjacent to the ventricles and in patients with recurrent disease.[15,48]

The optimal surgical management of malignant astrocytomas is controversial. Although the need for tissue diagnosis and the efficacy of debulking tumor for relief of mass effect in symptomatic patients is universally accepted, the effect of aggressive cytoreductive surgery on patient survival time is unclear. Radical tumor extirpation is intuitively logical and recommended by most neurosurgeons who treat children and adults; $[2,3,12,15,22,25,26,34,40,42,50,51]$ however, recent literature reviews, focusing primarily on adult patients, have failed to demonstrate a significant survival benefit of radical tumor resection.[10,30,35,41,44]

Previous reports in which the influence of the extent of tumor resection on malignant astrocytomas has been examined have been retrospective studies in which the influence of other prognostic variables or the vagaries of surgical and perioperative management have not been consistently considered.[35] The Children's Cancer Group (CCG)-945 trial is a prospective chemotherapy protocol for children with AA or GBM, which is stratified according to the extent of surgical resection. We report data we have 
obtained and review the current neurosurgical management of malignant astrocytomas of childhood and the influence of the extent of tumor resection on survival time.

\section{CLINICAL MATERIAL AND METHODS}

Between April 1985 and May 1990, 172 children between 2 and 21 years of age with histologically confirmed malignant brain astrocytomas were entered into and randomized according to the CCG-945 protocol. Children with intrinsic brainstem tumors were excluded from this study. Study entry occurred within 4 weeks of surgery and the institution's pathological diagnosis. After stratification according to the extent of tumor resection, patients were randomized to receive one or two chemotherapy regimens: eight-drugs-in-1-day ("8-in-1") chemotherapy[37] or a combination of prednisone, lomustine, and vincristine. No patient had received radiation or chemotherapy before study entry. All children underwent local field radiation therapy. Centralized pathological review of the disease was performed after study entry and randomization. The details of this protocol, including compliance with radiation and chemotherapy, have been previously reported.[17]

\section{Neurosurgical Management and Outcome}

The patient's neurosurgical report forms and operative records were reviewed to determine contemporary surgical management. The parameters examined included: tumor location, extent of tumor resection determined by surgical observation and postoperative computerized tomography (CT) or magnetic resonance (MR) imaging, operative instrumentation and technique, cerebrospinal fluid (CSF) diversion, and perioperative medications. The patient's postoperative neurological status and nonneurological complications present 1 week after surgery were documented.

The extent of tumor resection was qualitatively determined by the operating surgeon combining intraoperative observations with interpretation of postoperative contrast-enhanced CT or MR images. Resection was defined as biopsy ( $<10 \%$ resection) or as partial (10-50\%), subtotal (51-90\%), near-total (>90\%), or total resection. A tumor removal that constituted more than $90 \%$ was considered a radical resection.

\section{Statistical Analysis}

Overall survival (OS) and progression-free survival (PFS) times were the primary endpoints of the study. For patients whose therapy failed, PFS was defined as the time between randomization and tumor progression (clinical and/or radiographic) or death without progression. Patients who survived free of progression were censored as of the last date of contact. Analyses for this report were based on data available as of March 1997. Follow up of randomized patients has been outstanding. No patient was censored in the first 4 years and only two patients were censored in the first 6 years. Survival data were available for all children in the study; however, the analyses of OS and PFS times are restricted to patients with centrally reviewed and confirmed AA or GBM. The parameters analyzed included patient age, gender, and race, tumor location (superficial cerebral hematoma, deep or midline cerebrum, or posterior fossa), presence of metastatic disease, histological characteristics of the tumor (AA or GBM), extent of surgical resection, chemotherapy regimen, and the estimated volume of enhancing tumor on postoperative CT scans based on serial cross-sectional measurements $(>=1.5 \mathrm{~cm}$ compared with $<1.5$ cm maximum diameter).

Durations of PFS and OS were measured from the date of patient randomization to the date at which progressive disease was detected or the date of death or last contact as appropriate. Distributions of PFS 
and OS durations were estimated using the technique of Kaplan and Meier.[27] Standard errors of the Kaplan-Meier estimates were calculated as suggested by Peto and coworkers.[38,39] Comparisons between the distributions of PFS rates were made using a stratified Mantel-Haenszel statistic.[33] A Cox lifetable regression model[11] was used to evaluate simultaneously the prognostic importance of patient gender, race, and age, the extent of tumor resection, histological characteristics of the tumor, and the tumor's primary site of involvement within the group of children with a centrally reviewed pathological diagnosis of AA or GBM.

\section{RESULTS}

Clinical characteristics of the patient population are shown in Table 1 . The median age at study entry was 10.1 years (range 21 months-19 years). There were no statistical differences between patients randomized to the two treatment regimens on the basis of gender, race, age, primary tumor location, or the presence of metastatic disease at diagnosis.[17]

\section{Pathological Diagnosis}

Centralized neuropathological review confirmed the diagnosis of AA or GBM in 131 patients. The cases of three patients were not reviewed; 14 patients had other high-grade tumors, and the review diagnoses in 24 were consistent with low-grade gliomas. This report is based on the 131 patients in whom a confirmed diagnosis of AA or GBM was made.

\section{Operative Technique}

Operative techniques included craniotomy with a free bone flap in 59\% of cases, craniotomy with an osteoplastic flap in $22 \%$, burr hole in $10 \%$, and a craniectomy in $9 \%$ of cases. Craniectomy was only performed for infratentorial tumors. The dura was closed primarily in $79 \%$ of cases with an additional graft in 6\%; it was left partially or completely open in $14 \%$ of cases. The instrumentation commonly used during surgery included magnification (microscope in $36 \%$ of cases and loupes in $15 \%$ ), pin-fixation head holder (44\%), ultrasonic aspirator (42\%), diagnostic intraoperative ultrasound (18\%), and laser $(6 \%)$. Three drugs were frequently administered as perioperative medications: glucocorticosteroids (96\% of cases), mannitol (59\% of cases), and furosemide (19\% of cases).

\section{Tumor Location and Resection}

The vast majority of tumors were supratentorial in location: 63\% (83 tumors) in the superficial cerebral hemispheres, $28 \%$ (37 tumors) in the deep or midline cerebrum, and only $8 \%$ (11 tumors) in the posterior fossa (Tables 1 and 2). 


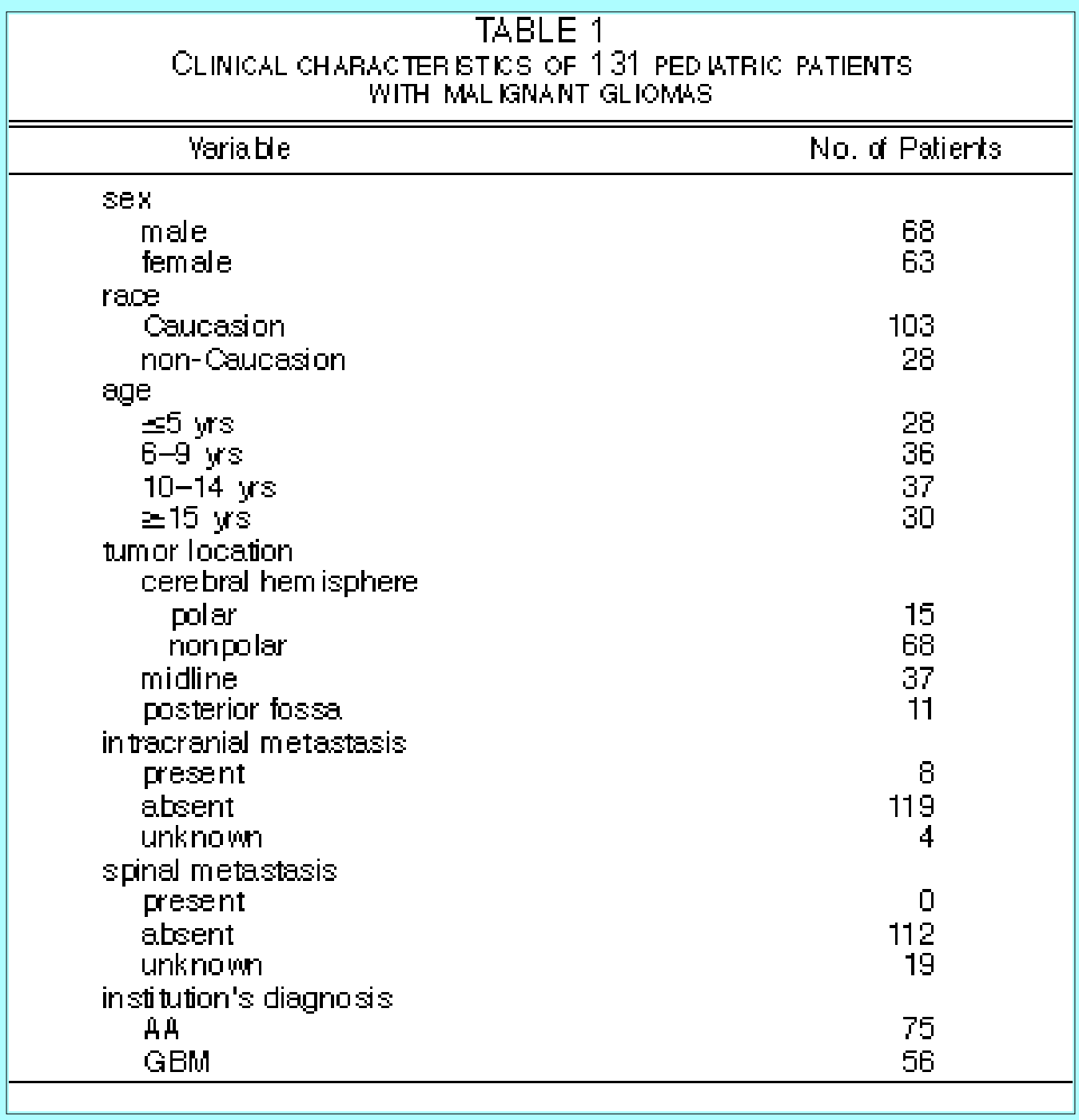

Among the cerebral hemisphere tumors, only 15 of 83 were polar in location (Table 1). A significant association was detected between the primary tumor site and the extent of surgical resection $(\mathrm{p}<0.0001)$. A radical resection ( $>90 \%$ ) was performed in $37 \%$ of the children; this constituted $49 \%$ of the tumors in the superficial hemisphere and $45 \%$ of the tumors in the posterior fossa compared with $8 \%$ of the midline tumors (Table 2).

\begin{tabular}{|c|c|c|c|c|}
\hline LOCATION AND EX & $\begin{array}{r}\text { Ti } \\
\text { F TUMOR Ri } \\
\text { MAL IGR }\end{array}$ & $\begin{array}{l}2 \\
\text { ION IN } 131 \\
\text { GLIOMAS }\end{array}$ & MTRIC PATIE & WITH \\
\hline $\begin{array}{c}\text { Extent of Surgical } \\
\text { Resection }\end{array}$ & $\begin{array}{c}\text { Ceretral } \\
\text { Hemisphere } \\
\text { (83 pab ents) }\end{array}$ & $\begin{array}{c}\text { Midine } \\
\text { (37 patients) }\end{array}$ & $\begin{array}{c}\text { Posterior } \\
\text { Fossa } \\
\text { [11 patients] }\end{array}$ & Total \\
\hline $\begin{array}{l}\text { biopsy }(\propto 10 \%) \\
\text { partial }(10-50 \%) \\
\text { subtotal }(51-90 \%) \\
\text { near-total }(>90 \%) \\
\text { total }(100 \%)\end{array}$ & $\begin{array}{c}5(6 \%) \\
11(13 \%) \\
26(31 \%) \\
38(46 \%) \\
3(4 \%)\end{array}$ & $\begin{array}{c}16(43 \%) \\
9(24 \%) \\
9(24 \%) \\
3(8 \%) \\
0\end{array}$ & $\begin{array}{l}1(9 \%) \\
1(9 \%) \\
4(36 \%) \\
5(45 \%) \\
0\end{array}$ & $\begin{array}{r}22 \\
21 \\
39 \\
46 \\
3\end{array}$ \\
\hline
\end{tabular}

\section{Diversion of CSF}

Tumor location was used to predict the need for both temporary and permanent CSF diversion. Preoperative or intraoperative ventriculostomy was performed in 19 patients; $22 \%$ of deep tumors 
required ventriculostomy compared with only $6 \%$ of superficial hemisphere neoplasms. Half of the deep tumors and $8 \%$ of the hemispheric astrocytomas ultimately required a permanent CSF shunt.

\section{Rates of Morbidity and Complications}

Improvement in preoperative neurological deficits and level of consciousness was seen in $36 \%$ and $34 \%$ of the children, respectively. At 1 week following surgery, new or increased deficits were present in $14 \%$ of the children, with $7 \%$ experiencing a diminished sensorium. Other postoperative complications were rare: infection, hematoma, and CSF fistula each occurred in $1.7 \%$ of cases. The rate of morbidity was unrelated to the operative technique, instrumentation, or extent of surgical resection.

\section{Progression-Free Survival and Overall Survival}

Both univariate and multivariate Cox lifetable regression analyses demonstrated that radical resection (> $90 \%$ ) was significant for improved PFS with a relative risk of 2.03 (95\% confidence interval 1.31-3.13) for less radical resection (Tables 3 and 4).

\begin{tabular}{|c|c|}
\hline \multicolumn{2}{|c|}{$\begin{array}{c}\text { TABLE } 3 \\
\text { RESULTS OF UN WARIATE COX LIFETABLE REGRESSION ANALYSS OF } \\
\text { CHARACTER ISTKS OF } 131 \text { PED ATRIC PATIENTS WITH MLL IGNANT GLIOMA }\end{array}$} \\
\hline Variatle & p Value \\
\hline $\begin{array}{l}\text { GBM ws AA } \\
\text { >90\% ws }=90 \% \text { tumor resection } \\
\text { "8 in 1" ws PCV chemotherapy' } \\
\text { patient age (yrs) }=5 \text { ws } 6-14 \text { ws }=15 \\
\text { non-Caucasian ws Caucasian } \\
\text { male ws female } \\
\text { cerebral hem isphere tumor ws Ta } \\
\text { midline tum or ws TO } \\
\text { posterior fossa tumor ws TQ }\end{array}$ & $\begin{array}{l}0.06 \\
0.03 \\
0.9 \\
0.9 \\
0.3 \\
0.04 \\
0.7 \\
0.2 \\
0.3\end{array}$ \\
\hline hustine, and vincr & othe \\
\hline
\end{tabular}

\begin{tabular}{|c|c|c|c|c|}
\hline \multicolumn{5}{|c|}{$\begin{array}{c}\text { TABLE } 4 \\
\text { RESULTS OF MULTWARIATE COX LIFETABLE REGRESSION ANALYSE OF } \\
\text { CHARACTER STKS OF } 131 \text { PED MTRIC PATIENTS WITH A MAL IGNANT GLIOMA }\end{array}$} \\
\hline Step & Variable Ertered & p Value & $\begin{array}{l}\text { Relabve } \\
\text { Risk }\end{array}$ & $\begin{array}{c}\mathrm{Cl} \\
95 \%\end{array}$ \\
\hline $\begin{array}{l}1 \\
2 \\
3\end{array}$ & $\begin{array}{c}>90 \% \text { ws }=90 \% \text { tumor resection } \\
\text { female ws male } \\
\text { GBM ws ÁA }\end{array}$ & $\begin{array}{l}\times 0.002 \\
\times 0.006 \\
\times 0.004\end{array}$ & $\begin{array}{l}2.03 \\
1.75 \\
1.85\end{array}$ & $\begin{array}{l}1.31-3.13 \\
1.17-2.60 \\
1.22-2.79\end{array}$ \\
\hline
\end{tabular}

This advantage was independent of patient gender, age, race, and tumor location. Within the hemispheric tumors, neither polar or nonpolar locations influenced the extent of tumor resection or the duration of PFS. Neither chemotherapy regimen demonstrated a superior PFS or OS rate.[17] The amount of residual disease $(<1.5 \mathrm{~cm}$ or $>=1.5 \mathrm{~cm}$ maximum dimension) measured on CT scanning was not significant for the PFS or OS rate. The pathological diagnosis of GBM and male gender were the only other significant variables affecting the length of PFS; both were associated with shorter PFS times.

Kaplan-Meier PFS curves for the extent of tumor resection are shown in Fig. 1. Figure 1 upper 
demonstrates the duration of PFS among all patients with malignant astrocytomas confirmed by centralized pathological review. The 5-year actuarial PFS rate was $35 \pm 7 \%$ following radical (>90\%) resection compared with $17 \pm 4 \%$ after lesser $(3 / 490 \%)$ resection $(\mathrm{p}=0.006)$. The graphs in Fig. 1 center and lower show the duration of PFS in children older than 3 years who have AAs and GBMs, respectively, without evidence of metastatic disease at diagnosis. Radical resection of an AA was associated with a $44 \pm 11 \%$ 5-year PFS rate compared with a $22 \pm 6 \% 5$-year PFS rate after a lesser $(3 / 4$ $90 \%)$ resection $(\mathrm{p}=0.055)$. Glioblastomas multiforme were associated with a $26 \pm 9 \% 5$-year PFS rate after radical resection compared with $4 \pm 3 \% 5$-year PFS after lesser resection $(\mathrm{p}=0.046)$.

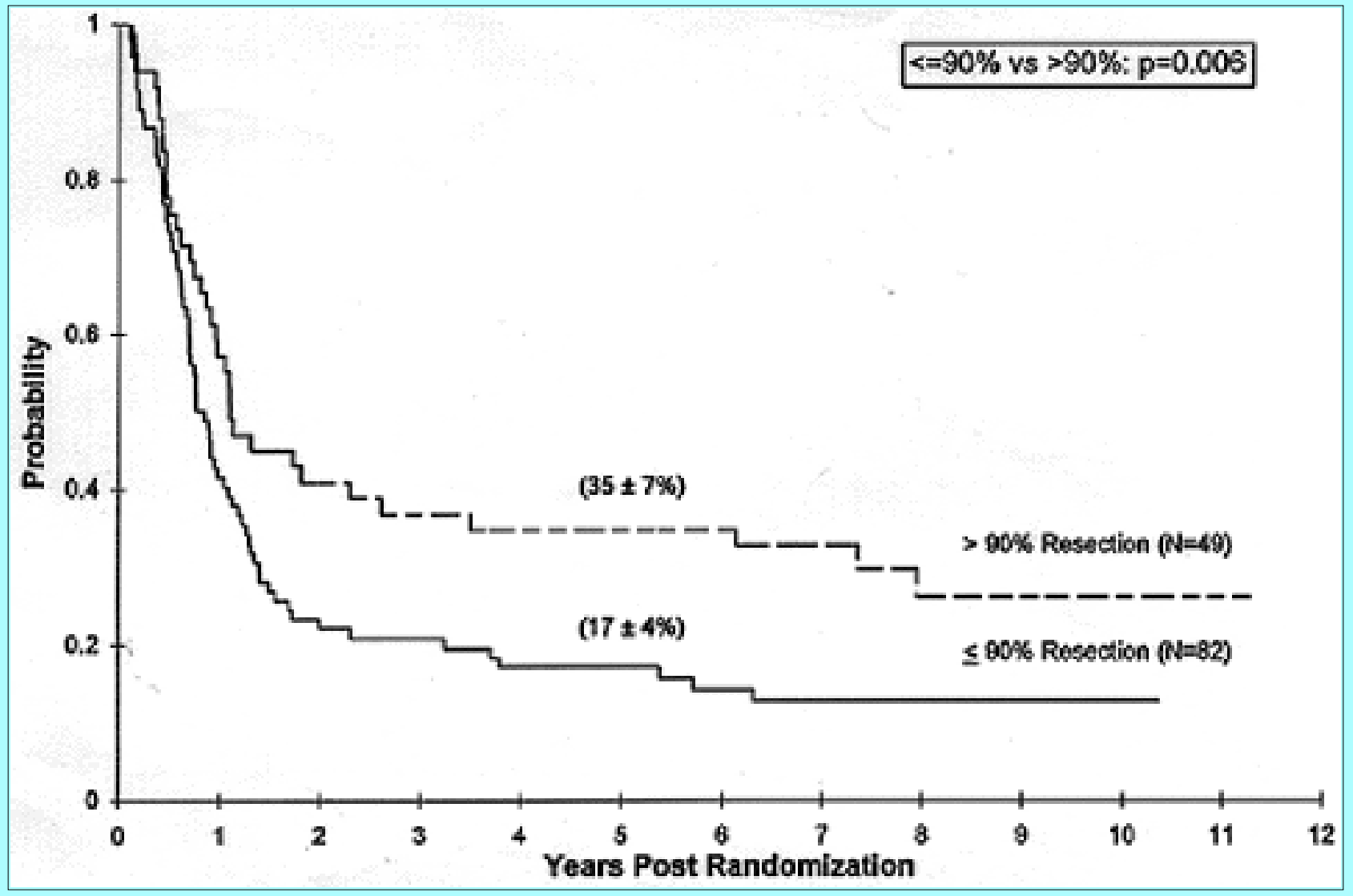



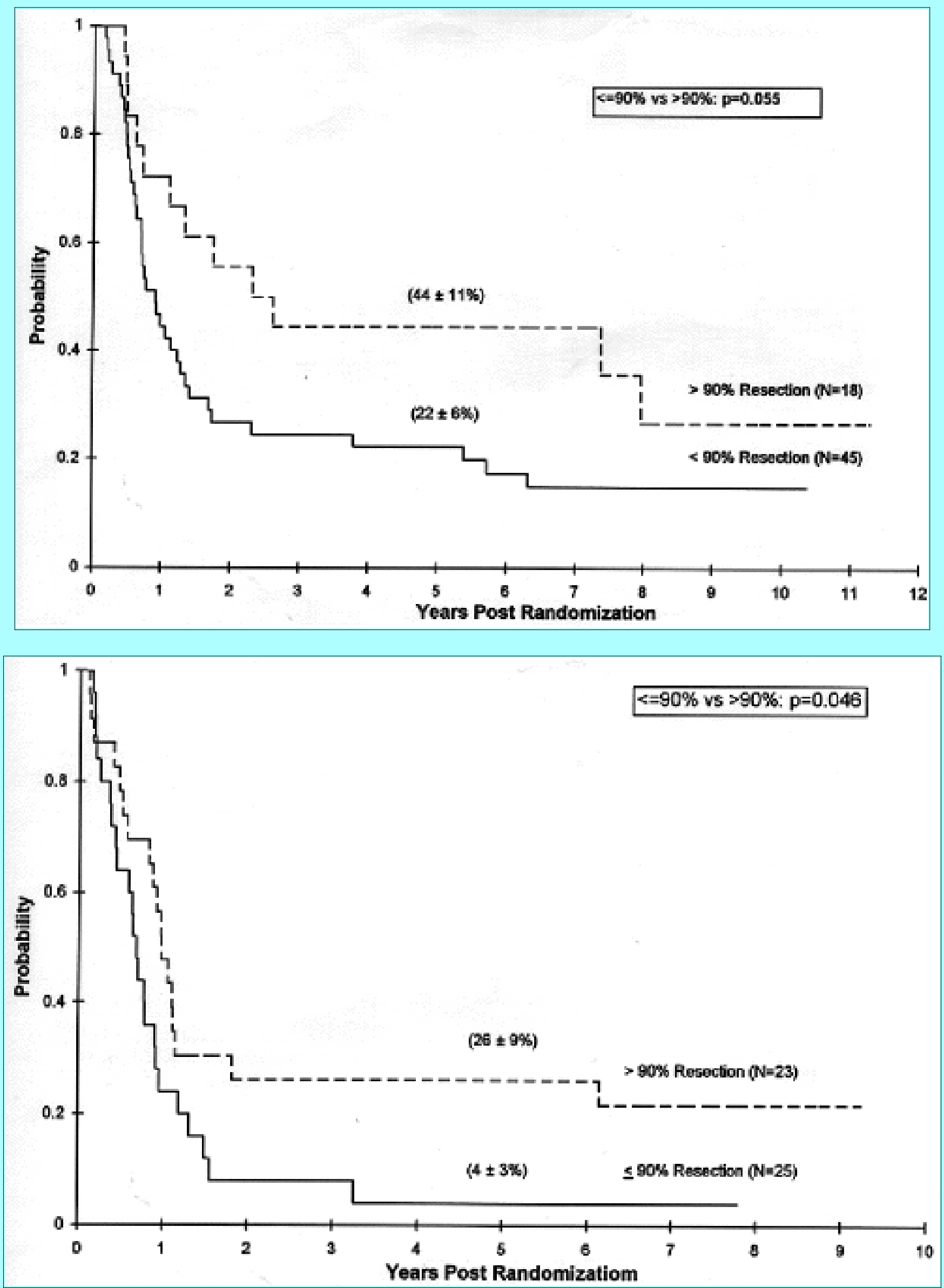

Fig. 1. Graphs displaying Kaplan-Meier PFS curves for the extent of tumor resection. Upper: Duration of PFS among all patients with AA or GBM confirmed by centralized 
pathological review. Center: Duration of PFS in patients with AA confirmed by centralized pathological review. Lower: Duration of PFS in all patients with GBM confirmed by centralized pathological review.

\section{DISCUSSION}

The benefits of radical surgical resection in patients with malignant astrocytomas are controversial. In their review of the literature Nazzaro and Neuwelt[35] noted that the majority of reports that have formed contemporary attitudes and recommendations on the value of surgical resection did not include statistical methods in study design or data analysis. The initial impetus for aggressive surgery was based on results from single-institution surgical series collected over a long period of time.[2,3,18,20,22,25,26,34,40,42] These retrospective reports showed a benefit of radical surgery on survival rates; however, the studies were prone to selection bias and failed to control for important characteristics including patient age and functional status and the tumor's location and histological characteristics, thereby rendering the studies' conclusions suspect. Further limitations on analysis of single-institution series are imposed by patterns of referral, incomplete follow-up information, and other missing data.

Cooperative group trials have primarily focused on the adult population; the impact of surgery on the length of survival has not been consistent among these trials. [8,19,44,49] The Brain Tumor Study Group found that patients who underwent gross-total resection with lobectomy lived longer than patients who underwent gross-total resection alone and that both of these groups had improved median survival times compared with patients who underwent only a biopsy.[19,49] Yet, when submitted to multivariate analysis, the extent of surgical resection lost its statistical significance. A review of serial Radiation Therapy Oncology Group trials found a patient survival advantage to maximum surgical resection, but failed to control for tumor location.[36] The Southwest Oncology Group did not find an advantage to surgery in a study that controlled for patient age and functional status and the pathological characteristics of the tumor.[16]

Only a small proportion of the overall population of adult patients with malignant astrocytomas has been entered into these studies. In addition, patients included in the studies had to be in good medical condition with a high Karnofsky Performance Scale score. Thus, study populations were inherently nonrepresentative of the spectrum of malignant astrocytomas. Maximum surgical resection prior to the trial of adjuvant therapy was the intent of the Brain Tumor Study Group,[19,49] but not of the protocols of the Radiation Therapy Oncology Group.[8] It is difficult to draw conclusions about cytoreductive surgery from these reports because of these inherent selection biases in the study populations as well as varying surgical philosophies and goals, selection of different prognostic variables for data analysis, and the construction of different statistical models.

Although the primary aim of the CCG-945 protocol was to examine the relative efficacy of two chemotherapy regimens, the prospectively collected data were stratified to allow additional analysis of the influence of surgery on PFS and OS. The most significant prognostic variable was radical surgical resection. The impact of cytoreductive surgery was observed for the entire study population, as well as for the subgroups of children with AAs and GBMs, as determined by either the institution's centralized review of the pathology. This analysis confirms the survival advantage for patients who undergo radical resection noted in a smaller previous CCG randomized study of children with malignant astrocytomas.[46]

Our patient population differs in several important respects from those included in previous studies. First, 
over half of the children with malignant astrocytomas anticipated to occur within the population base served by CCG institutions (CCG tumor registration files, 1994) were enrolled in this study. The 172 children represent the largest prospective study of malignant astrocytomas of childhood to date. This provides a diverse study group fully representative of the spectrum of these pediatric neoplasms.

Patient age and functional status are factors known to have a positive influence on the length of survival.[8,12,16,18-20,23,30,35,36,41,49] Our patients were not preselected for functional status and were representative of the entire pediatric cohort eligible for radiation therapy with similar numbers of patients in each hemidecade (Table 1). Reports of improved survival benefit for radical resection of malignant astrocytomas have been criticized for a basis in selecting out younger patients (among a population of adults) with better functional status.[10,30,35,41,50] There was no difference in survival rates among patients when age was compared by hemidecade $(3 / 45,6-14$, and $>=15$ years $)$. The biological characteristics of malignant astrocytomas treated by radiation and chemotherapy appear to be independent of age (excluding infancy) for the first two decades of life.

The third difference was that analysis of the extent of surgical resection was controlled for tumor location. Multivariate analysis demonstrated resection as an independent prognostic variable. Even among the hemispheric tumors, polar tumor location, as opposed to a nonpolar location, did not influence the extent of surgical resection or length of PFS. The final difference between our results and those of prior studies was the availability of detailed information concerning surgical management, morbidity, and complications.

Devaux, et al.,[12] reviewed the cases of 263 adults and children with malignant gliomas that were resected or obtained at biopsy by a single surgeon who used uniform techniques over a 5-year period. The analysis was stratified according to patient age, Karnofsky Performance Scale score, location of the tumor, its pathological characteristics, and the use of postoperative radiation therapy. The authors found a significant increase in median survival times for patients with GBMs, but not for those with AAs, who had undergone gross-total resection compared with biopsy. Similar to our patients, complications in those patients were few.

Winger and colleagues[50] reported an analysis of 285 adults with supratentorial AA consecutively treated at a regional cancer center. Although retrospective in design, their study is similar to the present report, in that it had the advantage of accruing all patients within a large population base; maintaining prospectively compiled, complete detailed records; and providing a uniform approach to the initial therapy. In these unselected patients, the extent of surgical resection, which was corrected for accessibility (that is, tumor location) was significant with respect to survival duration, independent of all other prognostic variables. Patients who had undergone gross-total resection lived significantly longer than those who had partial resections. In contrast to our patients, any degree of resection conferred a survival advantage over biopsy.

The prognostic importance of the extent of tumor resection has been previously correlated with the appearance of residual tumor on postoperative CT scanning.[32,52] We could not confirm this relationship in our patients. The study design, created in the early 1980s, did not stratify patients according to radiographic evidence of residual disease or provide for uniform central review of postoperative radiographs in all patients. Further complicating any attempt to analyze the impact of residual tumor observed on radiographs was the interval between surgery and postoperative imaging; the average time from resection to CT scanning was 6 days in our study. Authors of both animal[24] and 
clinical reports[6,7,9] have recommended performing CT scans within 2 to 4 days of surgery, to avoid spurious enhancement from the development of reactive neovascularity and breakdown of the blood-brain barrier in the tumor bed. Even with early scans, enhancement may occur in biopsy-negative resection margins of pediatric brain tumors only to disappear on subsequent studies.[47] Magnetic resonance imaging and computerized calculation of residual volume may prove to be a more precise method of documenting the extent of tumor resection and predicting outcome. This should be investigated in future protocols.

The management of our patients represents a cross section of current neurosurgical philosophy and technique. Neurosurgeons from 60 institutions who treat adults and children participated in this study. Radical resection was performed in half of the hemispheric tumors. The presence of new neurological deficits in $14 \%$ and diminished sensorium in $6 \%$ is higher than reported by institutional series.[9,12,28] This may be an artifact of collecting data early in the postoperative period, before patients could fully recover from the insults of surgery.

The correlation between the extent of tumor resection and PFS rates should prompt neurosurgeons to attempt radical resections in almost all children. Although the majority of operations used contemporary technology, including magnification, ultrasonic aspirators, and intraoperative ultrasound, many patients underwent a resection procedure that was technically no different from that used three decades ago. In the presence of significant residual tumor following an initial resection, a second operation must be considered. Ciric and coworkers[9] have shown that more than $85 \%$ of hemispheric astrocytomas are amenable to radical resection, with only a $3 \%$ morbidity rate. Routine use of stereotactic techniques[12,28] and functional monitoring[4] has permitted radical resection to be performed in deep tumors and eloquent brain locations with similar low rates of new deficits.

\section{CONCLUSIONS}

The outcome for children with malignant astrocytomas remains poor. Radical resection with preservation of neurological function is the optimal surgical treatment modality. Future use of stereotactic and functional techniques may extend radical cytoreductive surgery to deep tumors. The demonstration of a survival advantage to radical resection should prompt neurosurgeons to approach all malignant pediatric astrocytomas with the same aggressive attitude and operative techniques as they would use for benign, low-grade astrocytomas of childhood.[21,31]

\section{APPENDIX}

\section{Participating Investigators in the Children's Cancer Group}

Children's Cancer Group Operations Center, Arcadia, California, Principal Investigators: W. Archie Bleyer, M.D., Anita Khayat, Ph.D., Harland Sather, Ph.D., Mark Krailo, Ph.D., Jonathan Buckley, M.B.B.S., Ph.D., Daniel Stram, Ph.D., and Richard Sposto, Ph.D. (Grant No. CA 13539)

University of Michigan Medical Center, Ann Arbor, Michigan. Principal Investigator: Raymond Hutchinson, M.D. (Grant No. CA 02971)

University of California Medical Center, San Francisco, California. Principal Investigator: Katherine Matthay, M.D. (Grant No. CA 17829)

University of Wisconsin Hospital, Madison, Wisconsin. Principal Investigator: Paul Gaynon, M.D. 
(Grant No. CA 05436)

Children's Hospital \& Medical Center, Seattle, Washington. Principal Investigator: Ronald Chard, M.D. (Grant No. CA 10382)

Rainbow Babies \& Children's Hospital, Cleveland, Ohio. Principal Investigator: Susan Shurin, M.D. (Grant No. CA 20320)

Children's National Medical Center, Washington, D.C.. Principal Investigator: Gregory Reaman, M.D. (Grant No. CA 03888)

Children's Memorial Hospital, Chicago, Illinois. Principal Investigator: Edward Baum, M.D. (Grant No. CA 07431)

Children's Hospital of Los Angeles, Los Angeles, California. Principal Investigator: Jorge Ortega, M.D. (Grant No. CA 02649)

Children's Hospital of Columbus, Columbus, Ohio. Principal Investigator: Frederick Ruymann, M.D. (Grant No. CA 03750)

Columbia Presbyterian College of Physicians \& Surgeons, New York, New York. Principal Investigator: Sergio Piomelli, M.D. (Grant No. CA 03526)

Children's Hospital of Pittsburgh, Pittsburgh, Pennsylvania. Principal Investigator: Joseph Mirro, M.D. (Grant No. CA 36015)

Vanderbilt University School of Medicine, Nashville, Tennessee. Principal Investigator: John Lukens, M.D. (Grant No. CA 26270)

Doernbecher Memorial Hospital for Children, Portland, Oregon. Principal Investigator: Lawrence Wolff, M.D. (Grant No. CA 26044)

University of Minnesota Health Sciences Center, Minneapolis, Minnesota. Principal Investigator: William Woods, M.D. (Grant No. CA 07306)

University of Texas Health Sciences Center, San Antonio, Texas. Principal Investigator: Thomas Williams, M.D. (Grant No. CA 36004)

Children's Hospital of Philadelphia, Philadelphia, Pennsylvania. Principal Investigator: Anna Meadows, M.D. (Grant No. CA 11796)

Memorial Sloan-Kettering Cancer Center, New York, New York. Principal Investigator: Peter Steinherz, M.D. (Grant No. CA 42764)

James Whitcomb Riley Hospital for Children, Indianapolis, Indiana. Principal Investigator: Philip Breitfeld, M.D. (Grant No. CA 13809)

Hospital for Sick Children, Toronto, Canada. Principal Investigator: Mark Greenberg, M.B., CH.B. University of Utah Medical Center, Salt Lake City, Utah. Principal Investigator: Richard O'Brien, M.D. (Grant No. CA 10198) 
Strong Memorial Hospital, Rochester, New York. Principal Investigator: Harvey Cohen, M.D. (Grant No. CA 11174)

University of British Columbia, Vancouver, Canada. Principal Investigator: Christopher Fryer, M.D. (Grant No. CA 29013)

Children's Hospital Medical Center, Cincinnati, Ohio. Principal Investigator: Robert Wells, M.D., M.B., CH.B. (Grant No. CA 26126)

Harbor/UCLA \& Miller Children's Medical Center, Torrance/Long Beach, California. Principal Investigator: Jerry Finklestein, M.D. (Grant No. CA 14560)

University of California Medical Center (UCLA), Los Angeles, California. Principal Investigator: Stephen Feig, M.D. (Grant No. CA 27678)

University of Iowa Hospitals and Clinics, Iowa City, Iowa. Principal Investigator: Raymond Tannous, M.D. (Grant No. CA 29314)

Children's Hospital of Denver, Denver, Colorado. Principal Investigator: Lorrie Odom, M.D. (Grant No. CA 28851)

Mayo Clinic and Foundation, Rochester, Minnesota. Principal Investigator: Gerald Gilchrist, M.D. (Grant No. CA 28882)

University of Medicine \& Dentistry of New Jersey, Camden, New Jersey. Principal Investigator: Milton Donaldson, M.D.

Children's Mercy Hospital, Kansas City, Missouri. Principal Investigator: Maxine Hetherington, M.D.

University of Nebraska Medical Center, Omaha, Nebraska. Principal Investigator: Peter Coccia, M.D.

Wyler Children's Hospital, Chicago, Illinois. Principal Investigator: James Nachman, M.D.

M.D. Anderson Cancer Center, Houston, Texas. Principal Investigator: Beverly Raney, M.D.

New York University Medical Center, New York, New York. Principal Investigator: Aaron Rausen, M.D.

Children's Hospital of Orange County, Orange, California. Principal Investigator: Mitchell Cairo, M.D.

\section{References}

1. Al-Mefty O, Al-Rodham NRF, Phillips RL, et al: Factors affecting survival of children with malignant gliomas. Neurosurgery 20:416-420, 1987

2. Ammirati M, Vick N, Liao I, et al: Effect of the extent of surgical resection on survival and quality of life in patients with supratentorial glioblastomas and anaplastic astrocytomas. Neurosurgery 21:201-206, 1987

3. Artico M, Cervoni L, Celli P, et al: Supratentorial glioblastoma in children: a series of 27 surgically treated cases. Childs Nerv Syst 9:7-9, 1993 
4. Berger MS, Kincaid J, Ojemann GA, et al: Brain mapping techniques to maximize resection, safety, and seizure control in children with brain tumors. Neurosurgery 25:786-792, 1989

5. Burger PC, Vogel FS: Surgical Pathology of the Nervous System and Its Coverings, ed 2. New York: John Wiley \& Sons, 1982

6. Cairncross JG, Pexman JHW, Rathbone MP: Post-surgical contrast enhancement mimicking residual brain tumour. Can J Neurol Sci 12:75, 1985 (Letter)

7. Cairncross JG, Pexman JHW, Rathbone MP, et al: Postoperative contrast enhancement in patients with brain tumors. Ann Neurol 17:570-572, 1985

8. Chang HC, Horton J, Schoenfeld D, et al: Comparison of postoperative radiotherapy and combined postoperative radiotherapy and chemotherapy in the multidisciplinary management of malignant glioma. A Joint Radiation Therapy Oncology Group and Eastern Cooperative Oncology Group study. Cancer 52:997-1007, 1983

9. Ciric I, Ammirati M, Vick N, et al: Supratentorial gliomas: surgical considerations and immediate postoperative results. Gross total resection versus partial resection. Neurosurgery 21:21-26, 1987

10. Coffey RJ, Lunsford LD, Taylor FH: Survival after stereotactic biopsy of malignant gliomas. Neurosurgery 22:465-473, 1988

11. Cox DR: Regression models and life tables. J Roy Stat Soc Br 34:187-220, 1972

12. Devaux BC, O'Fallon JF, Kelly PJ: Resection, biopsy, and survival in malignant glial neoplasms. A retrospective study of clinical parameters, therapy, and outcome. J Neurosurg 78:767-775, 1993

13. Dohrmann GJ, Farwell JR, Flannery JT: Astrocytomas in childhood: a population-based study. Surg Neurol 23:64-68, 1985

14. Dohrmann GJ, Farwell JR, Flannery JT: Glioblastoma multiforme in children. J Neurosurg 44:442-448, 1976

15. Dropcho EJ, Wisoff JH, Walker RW, et al: Supratentorial malignant gliomas in childhood: a review of fifty cases. Ann Neurol 22:355-364, 1987

16. Eyre HJ, Quagliana JM, Eltringham JR, et al: Randomized comparisons of radiotherapy and CCNU versus radiotherapy, CCNU plus procarbazine for the treatment of malignant gliomas following surgery. A Southwest Oncology Group Report. J Neurooncol 1:171-177, 1983

17. Finlay JL, Boyett JM, Yates AJ, et al: Randomized phase III trial in childhood high-grade astrocytoma comparing vincristine, lomustine, and prednisone with the eight-drugs-in-1-day regimen. J Clin Oncol 13:112-123, 1995

18. Garcia DM, Fulling KH, Marks JE: The value of radiation therapy in addition to surgery for astrocytomas of the adult cerebrum. Cancer 55:919-927, 1985

19. Green SB, Byar DP, Walker MD, et al: Comparisons of carmustine, procarbazine, and high-dose methylprednisolone as additions to surgery and radiotherapy for the treatment of malignant glioma.

Cancer Treat Rep 67:121-132, 1983 
20. Hirakawa K, Suzuki K, Ueda S, et al: Multivariate analysis of factors affecting postoperative survival in malignant astrocytoma. Importance of DNA quantification. J Neurooncol 2:331-340, 1984

21. Hirsch JF, Sainte Rose C, Pierre-Kahn A, et al: Benign astrocytic and oligodendrocytic tumors of the cerebral hemispheres in children. J Neurosurg 70:568-572, 1989

22. Hoppe-Hirsch E, Hirsch JF, Lellouch-Tubiana A, et al: Malignant hemispheric tumors in childhood. Childs Nerv Syst 9:131-135, 1993

23. Hoshino T: A commentary on the biology and growth kinetics of low-grade and high-grade gliomas. J Neurosurg 61:895-900, 1984

24. Jeffries BF, Kishore PRS, Singh KS, et al: Contrast enhancement in the postoperative brain. Radiology 139:409-413, 1982

25. Jelsma R, Bucy PC: Glioblastoma multiforme. Its treatment and some factors affecting survival. Arch Neurol 20:161-171, 1969

26. Jelsma R, Bucy PC: Treatment of glioblastoma multiforme of the brain. J Neurosurg 27:388-400, 1967

27. Kaplan EL, Meier P: Nonparametric estimation from incomplete observations. J Am Stat Assoc 53:457-481, 1958

28. Kelly PJ: Stereotactic biopsy and resection of thalamic astrocytomas. Neurosurgery 25:185-195, 1989

29. Kramer S, Meadows AT, Jarrett P, et al: Incidence of childhood cancer: experience of a decade in a population-based registry. J Natl Cancer Inst 70:49-55, 1983

30. Kreth FW, Warnke PC, Scheremet R, et al: Surgical resection and radiation therapy versus biopsy and radiation therapy in the treatment of glioblastoma multiforme. J Neurosurg 78:762-766, 1993

31. Laws ER Jr, Taylor WF, Clifton MB, et al: Neurosurgical management of low-grade astrocytoma of the cerebral hemispheres. J Neurosurg 61:665-673, 1984

32. Levin VA, Hoffman WF, Heilbron DC, et al: Prognostic significance of the pretreatment CT scan on time to progression for patients with malignant gliomas. J Neurosurg 52:642-647, 1980

33. Mantel N: Evaluation of survival data and two new rank order statistics arising in its consideration. Cancer Chemother Rep 50:163-170, 1966

34. Marchese MJ, Chang CH: Malignant astrocytic gliomas in children. Cancer 65:2771-2778, 1990

35. Nazzaro JM, Neuwelt EA: The role of surgery in the management of supratentorial intermediate and high-grade astrocytomas in adults. J Neurosurg 73:331-344, 1990

36. Nelson DF, Nelson JS, Davis DR, et al: Survival and prognosis of patients with astrocytoma with atypical or anaplastic features. J Neurooncol 3:99-103, 1985

37. Pendergrass TW, Milstein JM, Geyer JR, et al: Eight drugs in one day chemotherapy for brain tumors: experience in 107 children and rationale for preradiation chemotherapy. J Clin Oncol 
38. Peto R, Pike MC, Armitage P, et al: Design and analysis of randomized clinical trials requiring prolonged observation of each patient. I. Introduction and design. Br J Cancer 34:585-612, 1976

39. Peto R, Pike MC, Armitage P, et al: Design and analysis of randomized clinical trials requiring prolonged observation of each patient. II. Analysis and examples. Br J Cancer 35:1-39, 1977

40. Phuphanich S, Edwards MSB, Levin VA, et al: Supratentorial malignant gliomas of childhood. Results of treatment with radiation therapy and chemotherapy. J Neurosurg 60:495-499, 1984

41. Quigley MR, Maroon JC: The relationship between survival and the extent of the resection in patients with supratentorial malignant gliomas. Neurosurgery 29:385-389, 1991

42. Ransohoff J, Kelly P, Laws E: The role of intracranial surgery for the treatment of malignant gliomas. Semin Oncol 13:27-37, 1986

43. Russell DS, Rubinstein LJ: Pathology of Tumours of the Nervous System, ed 4. Baltimore: Williams \& Wilkins, 1977

44. Salcman M: Survival in glioblastoma: historical perspective. Neurosurgery 7:435-439, 1980

45. Silverberg E: Cancer statistics, 1986. CA Cancer J Clin 36:9-23, 1986

46. Sposto R, Ertel IJ, Jenkin RDT, et al: The effectiveness of chemotherapy for treatment of high grade astrocytoma in children: results of a randomized trial. A report from the Childrens Cancer Study Group. J Neurooncol 7:165-177, 1989

47. Steinbok P, Poskitt K, Cochrane DD, et al: Early computed tomographic scanning after resection of brain tumor in children. Childs Nerv Syst 7:16-20, 1991

48. Vertosick FT Jr, Selker RG: Brain stem and spinal metastasis of supratentorial glioblastoma multiforme: a clinical series. Neurosurgery 27:516-522, 1990

49. Walker MD, Green SB, Byar DP, et al: Randomized comparisons of radiotherapy and nitrosoureas for the treatment of malignant glioma after surgery. N Engl J Med 303:1323-1329, 1980

50. Winger MJ, Macdonald DR, Cairncross JG: Supratentorial anaplastic gliomas in adults. The prognostic importance of extent of resection and prior low-grade glioma. J Neurosurg 71:487-493, 1989

51. Wisoff JH: Tumors of the cerebral hemispheres, in Cheek WR, Marlin AE, McClone DG, et al (eds): Pediatric Neurosurgery: Surgery of the Developing Nervous System, ed 3. Philadelphia: WB Saunders, 1994, pp 392-402

52. Wood JR, Green SB, Shapiro WR: The prognostic importance of tumor size in malignant gliomas: a computed tomographic scan study by the Brain Tumor Cooperative Group. J Clin Oncol 6:338-343, 1988

Manuscript received December 23, 1996.

Accepted in final form February 16, 1998. 
Dr. Boyett and Mr. Li were supported by Grant No. CA 21765 from the Division of Cancer Treatment, National Cancer Institute, and by the American Lebanese Syrian Associated Charities.

Current address for Dr. Berger: University of California at San Francisco, San Francisco, California. Address reprint requests to: Jeffrey H. Wisoff, M.D., Children's Cancer Group, P.O. Box 60012, Arcadia, California 91066-6012. 